\title{
Effects of random atomic disorder on the magnetic stability of graphene nanoribbons with zigzag edges
}

\author{
K. E. Çakmak, A. Altıntaş, and A. D. Güçlü \\ Department of Physics, Izmir Institute of Technology, IZTECH, TR35430 Izmir, Turkey
}

(Received 19 April 2018; revised manuscript received 31 July 2018; published 17 September 2018)

\begin{abstract}
We investigate the effects of randomly distributed atomic defects on the magnetic properties of graphene nanoribbons with zigzag edges using an extended mean-field Hubbard model. For a balanced defect distribution among the sublattices of the honeycomb lattice in the bulk region of the ribbon, the ground-state antiferromagnetism of the edge states remains unaffected. By analyzing the excitation spectrum, we show that while the antiferromagnetic ground state is susceptible to single spin-flip excitations from edge states to magnetic defect states at low defect concentrations, its overall stability is enhanced with respect to the ferromagnetic phase.
\end{abstract}

DOI: 10.1103/PhysRevB.98.115428

\section{INTRODUCTION}

The possibility to induce magnetism in graphene through sublattice engineering of the honeycomb lattice can potentially lead to a new class of spintronic and magnetic nanodevices [1-11]. Indeed, although pure graphene is not expected to be magnetic, Lieb's [12] bipartite lattice theorem for the Hubbard model predicts a finite total spin related to breaking of the sublattice symmetry. This broken symmetry can happen, for instance, at the zigzag edges of a graphene nanostructure [13-27] or around an atomic defect [29-42], resulting in magnetized localized states.

In zigzag graphene nanoribbons (ZGNRs), as the opposite edge atoms belong to opposite sublattices, one expects antiferromagnetically coupled zigzag-localized edge states with zero total spin. The induced magnetic behavior is predicted by several theoretical models, including density functional theory [14,15,36,37], the mean-field approximation of the Hubbard model [13,20,22,23,31], exact diagonalization [11,27], and quantum Monte Carlo simulation [25]. However, on the experimental side [43-53], the direct observation of magnetism in graphene nanoribbons is still lacking, most likely due to limited control over edge structure. Recently, a semiconductor-to-metal transition as a function of ribbon width was observed in nanotailored graphene ribbons with zigzag edges [26], attributed to a magnetic phase transition from the antiferromagnetic (AFM) configuration to the ferromagnetic (FM) configuration, raising hopes for the fabrication of graphene-based spintronic and magnetic storage devices. Possible theoretical explanations for the observed AFM to FM transition in ZGNRs include doping [54-56] and formation of electron-hole puddles through long-range Coulomb impurities [20].

Atomic defects also have significant influence on the magnetic properties of graphene, as has been shown before in several theoretical works [29-38,40]. In particular, vacancies and adatoms are expected to create midgap states [28] and to lead to magnetic moments localized around the defects with rich many-body effects $[29,38]$. Recently, the existence of magnetism in graphene by using hydrogen atoms was observed [33] and another direct experimental evidence of the magnetism in graphene due to single atomic vacancy in graphene was detected by using scanning tunneling microscope [40]. An open question is the effect of the induced magnetic moment by a random distribution of atomic defects on the stability of the antiferromagnetic phase of the ZGNR.

In this work, we investigate the magnetic phases of ZGNRs containing 10010 atoms with randomly distributed atomic defects using mean-field Hubbard calculations. We show that the atomic defects stabilize the antiferromagnetic phase of the ZGNRs. Our finding suggests that it should be easier to directly observe and control magnetism in ZGNRs through the generation of randomly distributed atomic defects (vacancies or adatoms) on the bulk region of the ribbon.

The paper is organized as follows. In Sec. II, we introduce the mean-field Hubbard Hamiltonian and the vacancy model that we use in the numerical calculations. In Sec. III, we present the numerical results showing the robustness of edge antiferromagnetism against disorder and demonstrate that the AFM stability increases with increasing defect concentration. Section IV contains a brief summary.

\section{MODEL AND METHOD}

Our starting point is the tight-binding model for $p_{z}$ orbitals, where $s, p_{x}$, and $p_{y}$ orbitals are disregarded as they mainly contribute to the mechanical stability of graphene. Atomic defects are modeled as randomly distributed vacancies, where the $p_{z}$ orbitals are simply removed from the honeycomb lattice. The vacancy mimics the hybridization of the corresponding $p_{z}$ orbital with a hydrogen adatom. We note that (i) possible lattice distortion near the defect sites which could suppress the magnetization [30] are neglected, (ii) zigzag edges are assumed to be properly hydrogen passivated and free of edge reconstruction [18,57,58], and (iii) atomic defects are assumed to be in the nanoribbon's bulk region only. We consider defect concentrations of $1 \%-5 \%$ of the total number of atoms. Moreover, within the extended mean-field Hubbard model used here, possible correlation effects on magnetic properties are neglected [11,27,29]. Our model Hamiltonian 
(a)

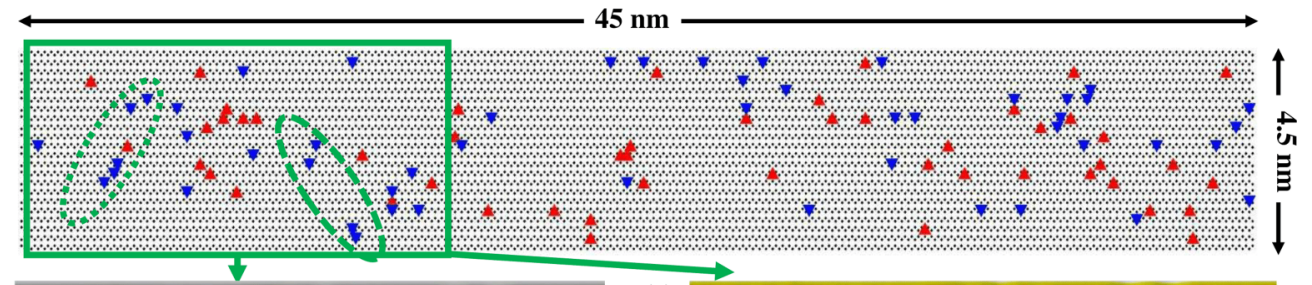

(b)

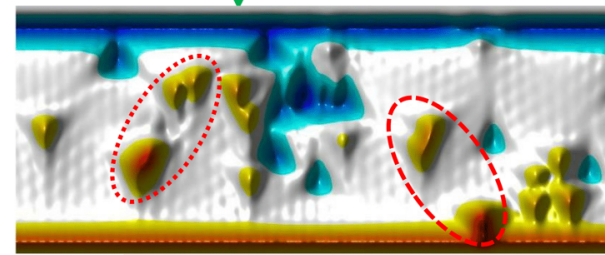

(d)

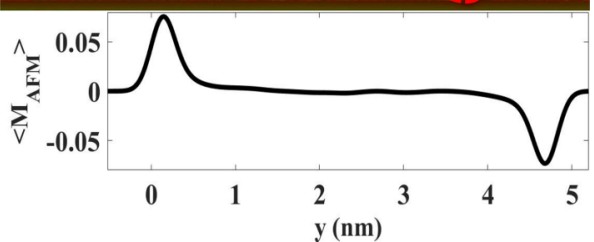

(c)

(e)

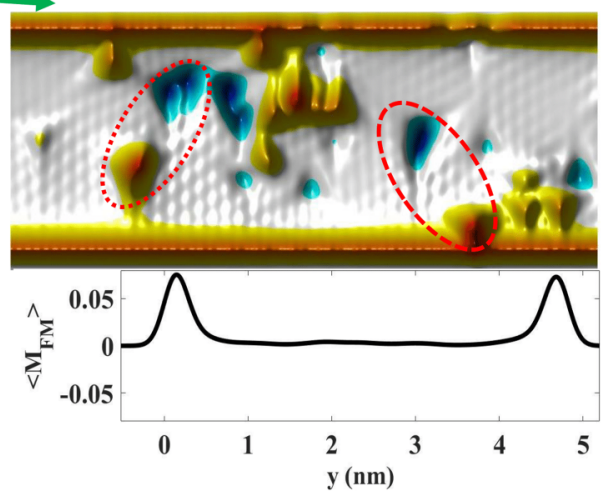

FIG. 1. (a) Graphene nanoribbon lattice structure with 10010 atoms and randomly generated $1 \%$ defect concentration equally distributed within the A sublattice (downward pointed triangles) and the B sublattice (upward pointing triangles). (b) AFM (ground state) and (c) FM spin density profile of a portion of the ribbon. (d) and (e) Average magnetization along the ribbon length for the AFM and FM phases.

is given by

$$
\begin{aligned}
H_{\mathrm{MFH}}= & \sum_{i j \sigma}\left(t_{i j} c_{i \sigma}^{\dagger} c_{j \sigma}+\text { H.c. }\right) \\
& +U \sum_{i \sigma}\left(\left\langle n_{i \sigma}\right\rangle-\frac{1}{2}\right) n_{i \bar{\sigma}}+\sum_{i j} V_{i j}\left(\left\langle n_{j}\right\rangle-1\right) n_{i} .
\end{aligned}
$$

The first term is for the tight-binding approximation where the $t_{i j}$ are the hopping parameters and are taken to be $t_{\mathrm{nn}}=$ $-2.8 \mathrm{eV}$ for the nearest neighbors and $t_{\mathrm{nnn}}=-0.1 \mathrm{eV}$ for the second nearest neighbors $[59,60]$. The creation, $c_{i \sigma}^{\dagger}$, and annihilation, $c_{i \sigma}$, operators create and annihilate an electron at the $i$ th orbital with spin $\sigma$, respectively. The term $\left\langle n_{i \sigma}\right\rangle$ corresponds to the expectation values of electron densities. The second and third terms are on-site and long-range Coulomb interaction terms, respectively. The on-site Coulomb potential $U$ is taken to be $16.522 / \kappa \mathrm{eV}$, where $\kappa=6$ is an effective dielectric constant. The long-range interaction parameters $V_{i j}$ are taken to be $8.64 / \kappa \mathrm{eV}$ and $5.33 / \kappa \mathrm{eV}$ for the first two neighbors and $1 / d_{i j} \kappa$ for distant neighbors [61]. However, unlike for ZGNRs in the presence of long-range disorder [20], the effect of long-range Coulomb interactions is found to be negligible in the presence of atomic defects considered in current work. This is because the total charge distribution is nearly constant over lattice sites since there is no potential variation and the system is taken to be charge neutral.

We consider 45-nm-long and 4.5-nm-wide ZGNRs consisting of 10010 atoms with various defect configurations. Figure 1(a) shows a ZGNR configuration with $1 \%$ of defects that are randomly and equally distributed among the two sublattices of the honeycomb lattice. The downward pointing (blue color online) and upward pointing (red color online) triangles correspond to sublattice A-site and sublattice Bsite vacancies, respectively. The self-consistent Hubbard calculations were performed within different $S_{z}=\left(N_{\uparrow}-N_{\downarrow}\right) / 2$ subspaces to find the overall ground state. As one may suspect a competition between the AFM and FM states [20], we have scanned the $0 \leqslant S_{z} \leqslant 130$ values, with a focus around the AFM state $S_{z}=0$ and the FM state $S_{z}=N_{\text {edge }} / 2$, where the number of edge states is given by $N_{\text {edge }}=138$ for the clean structure. For each value $S_{z}$, the self-consistent calculations were repeated with different initial density matrices to ensure the convergence to the global energy minimum.

\section{RESULTS}

Figures 1(b) and 1(c) show the spin densities of a portion of the ribbon, for the lowest-energy AFM and FM states, respectively. Despite the inclusion of long-range electron interactions and second nearest-neighbor hoppings, the mean-field solution to the Hubbard model leads to the $S_{z}=0$ ground state in all our calculations with equally distributed defects among the two sublattices, in agreement with Lieb's theorem. Indeed, in Fig. 1(b), the A-site and B-site defects lead to spin-up (red color online) and spin-down (blue color online) magnetic moments, respectively, in agreement with previous quantum Monte Carlo calculations for pristine graphene [29]. On the other hand, the spin density distribution for the lowest FM state is harder to predict since it is not a ground state consistent with Lieb's theorem. Interestingly, the edge ferromagnetism of the $S_{z}=73$ state remains robust [see Fig. 1(c)] and the bulk atoms have a zero average magnetization as shown in Figs. 1(d) and 1(e). This simple observation has an important consequence on the stability of the AFM phase with respect to the FM phase: For the FM phase, the magnetization of the defects nearby edge atoms is strictly dictated by the strong magnetization of the edges, locally obeying Lieb's theorem. Hence, far from the edges, one must encounter sublattice spin frustrations where Lieb's theorem cannot be locally satisfied, costing energy. For instance, for the AFM state where the Lieb's theorem is globally satisfied, the A-site defects in the 


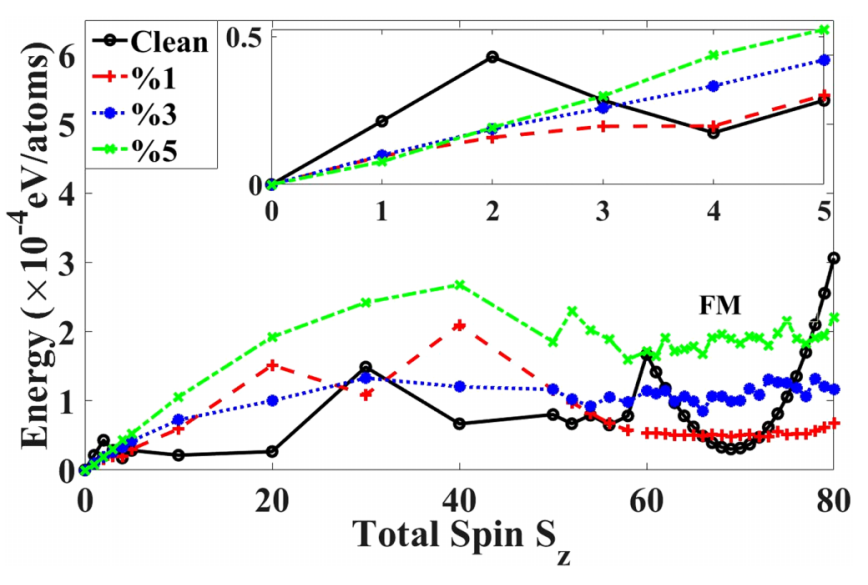

FIG. 2. Mean-field energy per atom as a function of total spin $S_{z}$ for the clean and the $1 \%, 3 \%$, and $5 \%$ defect concentration cases. For the clean case, the ground state is AFM edges with $S_{z}=0$, and the FM phase occurs at $S_{z}=69$. The FM-AFM gap increases with increasing defect concentration.

encircled areas in Fig. 1(b) are ferromagnetically coupled to each other, whereas their coupling is antiferromagnetic in Fig. 1(c). Our calculations show that such local violation of Lieb's theorem only occurs among defect sites and never between an edge and a defect site.

As discussed above, local violation of Lieb's theorem in the bulk region of the FM phase costs energy. A striking consequence of the energy cost is an increased stability of the AFM phase with respect to the FM phase. Figure 2 shows the energy per atom of different magnetic states $S_{z}$ with respect to the AFM ground state, for various defect concentrations up to $5 \%$. For the clean structure, the FM phase is at $S_{z}=N_{\text {edge }} / 2=69$ and the FM-AFM gap is $3.041 \times 10^{-5} \mathrm{eV} /$ atom. We note that, due to the finite-size effects, we see that states with $S_{z}=10$ and $S_{z}=20$ have lower energy than $S_{z}=69$ for the clean case. Moreover, As the defect concentration is increased, the FM-AFM gap increases, reaching $1.6 \times 10^{-4} \mathrm{eV}$ for the $5 \%$ of defects. Strikingly, the gap increase with respect to the AFM phase occurs not only for the FM phase but also for most other $S_{z}$ states. However, in the vicinity of $S_{z}=0$ (see the inset), i.e., for single and few spin flips, the energy cost is decreased at $1 \%$ defect concentrations, but then increases slightly with increasing numbers of defects. This reflects the fact that for low defect concentrations it is easier to flip an edge spin by moving it into a defect state than into the opposite edge. We note that similar behaviors were observed for other randomly generated defect concentrations and a statistical analysis is presented below in Fig. 4.

Figure 3 shows the mean-field density of states (DOS) for the AFM ground state, for different concentrations considered in Fig. 1. The solid lines represent the total DOS, whereas the dotted and dashed lines represent the contributions from edge and defect atoms (more precisely, atoms neighboring the defects and vacancies) to the DOS, respectively. For the clean nanoribbon, there is an AFM gap opening due to electronelectron interaction between the edge states, which is about $0.2143 \mathrm{eV}$, roughly corresponding to the energy required to flip a single spin. As the defect concentration is increased to $1 \%$, there is an increase of midgap state density [28,29] and the AFM gap is decreased to $0.1176 \mathrm{eV}$. This is consistent with the single spin flips in the vicinity of $S_{z}=0$ discussed in Fig. 1. When the concentration of defects is increased further [see Figs. 3(c) and 3(d)], the AFM gap starts increasing slightly. This change of behavior reflects the fact that for higher numbers of defects the magnetic coupling between
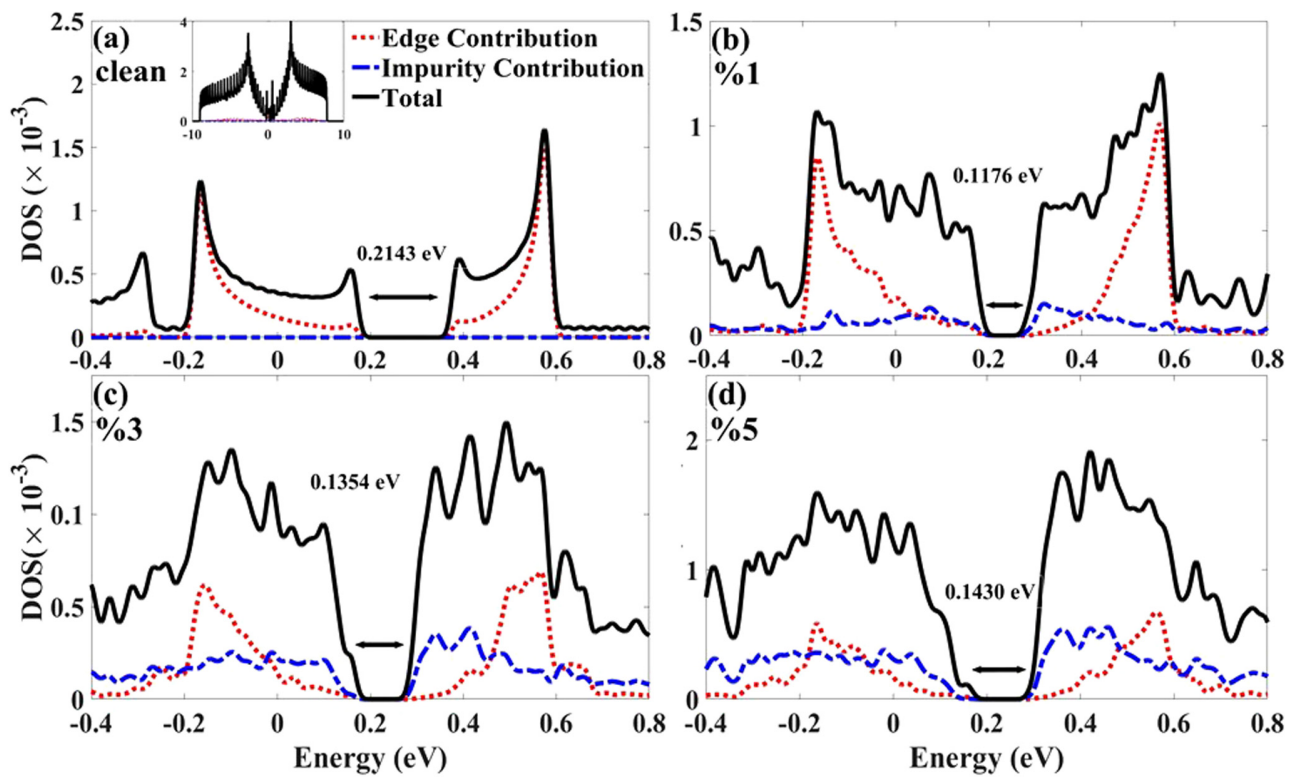

FIG. 3. Mean-field DOS for the AFM phase for the (a) clean, (b) $1 \%$ concentration, (c) $3 \%$ concentration, and (d) $5 \%$ defect concentration cases. Edge and defect state contributions are plotted with dotted and dot-dashed lines, respectively. Energy gap values of the total DOS are given for each case. 

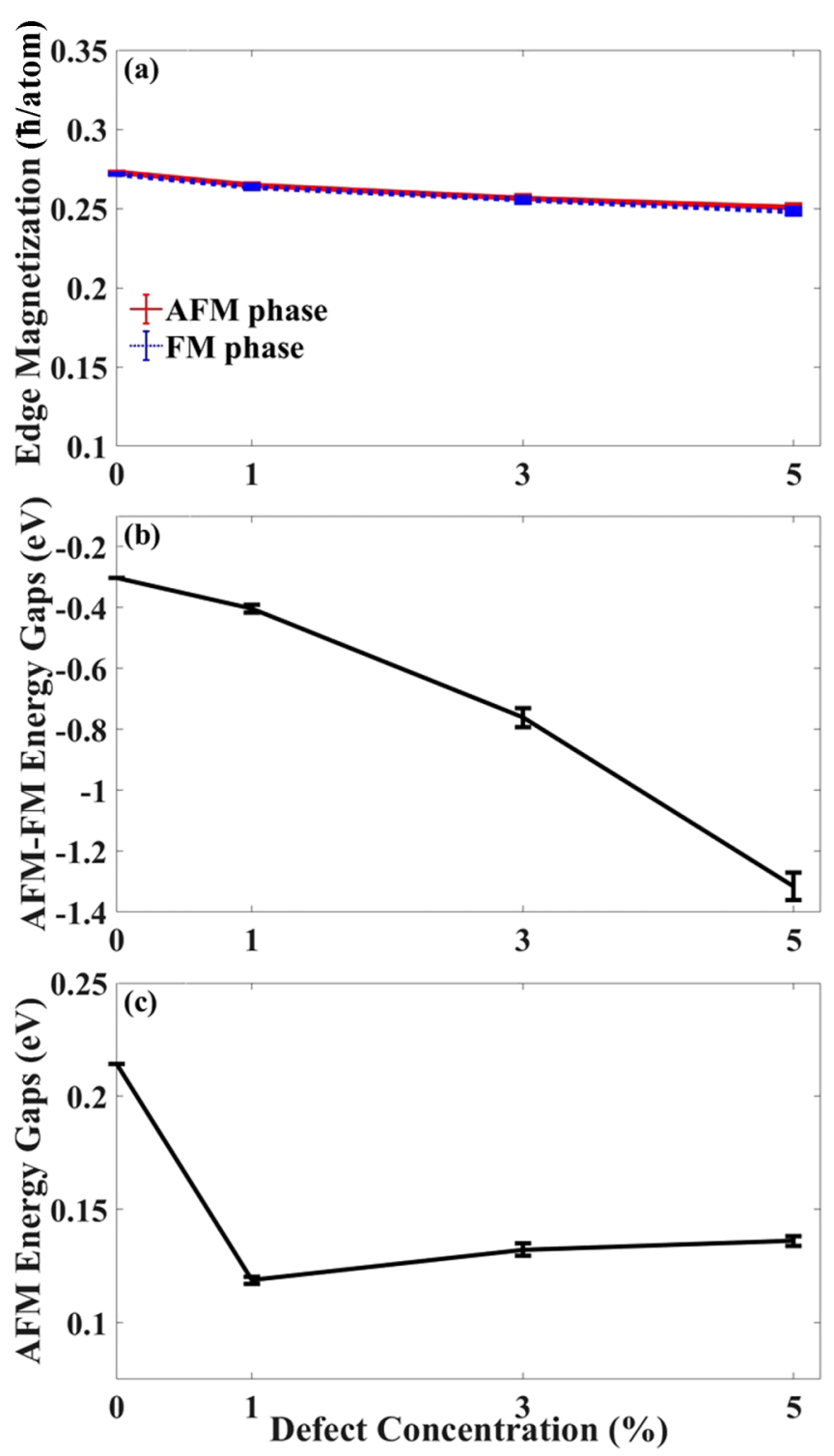

FIG. 4. Average (a) single edge magnetization, (b) AFM-FM energy gap, and (c) AFM energy gap, over ten randomly generated disorder configurations, as a function of defect concentration. Bars and symbols represent standard errors over the ten random configurations.

the defects is enhanced on average, stabilizing the magnetic configuration and making the spin flips harder. However, we note that the AFM-FM gap monotonically increases with increasing midgap states due to the local violation of Lieb's theorem, as discussed earlier.

Up to this point, the results presented were obtained for particular randomly generated defect configurations. For a statistical analysis of our results, we have repeated our calculations for ten randomly generated configurations at $1 \%$, $3 \%$, and $5 \%$ defect concentrations. We have observed similar behavior in all the disorder configurations and the results are presented in Fig. 4 as a function of defect concentration. The average magnetization of edge atoms for the AFM and FM phases, shown in Fig. 4(a), decreases slightly with increasing defect concentration. The difference between the AFM and FM edge magnetizations remains negligible (within the error bars), consistent with Figs. 1(d) and 1(e). On the other hand, Fig. 4(b) shows that the AFM-FM gap rapidly decreases on average with a small error bar, clearly demonstrating an increased stability of the AFM phase with respect to the FM phase. Finally, the average AFM gap shown in Fig. 4(c), indicating the energy cost for a single spin flip, systematically undergoes a decrease at lower concentrations and then keeps slowly increasing at concentrations higher than $1 \%$ due to a more stable magnetic lattice formed by defects.

\section{CONCLUSION}

In summary, effects of randomly distributed atomic defects on the stability of magnetic phases of a zigzag-edged graphene nanoribbon were investigated using a mean-field Hubbard approach. For an equal distribution of atomic defects among the two sublattices of the honeycomb lattice, the coupling between the two edges remains antiferromagnetic with $S_{z}=$ 0 . At lower defect concentrations $(\leqslant 1 \%)$, the energy of single spin flips from the antiferromagnetic ground state is decreased due to possible electron transfer from edges to defect states. However, we show that the AFM-FM energy gap remains well protected and is enhanced as a function increasing defect concentration. The increased stability of the AFM-FM gap by controlling defect concentrations opens up new possibilities for spintronic and magnetic nanodevice applications.

\section{ACKNOWLEDGMENTS}

This work was supported by the Scientific and Technological Research Council of Turkey (TUBITAK) under the 1001 Grant Project, Grant No. 114F331. The numerical calculations reported in this work were partially performed at TUBITAK ULAKBIM, High Performance and Grid Computing Center (TRUBA resources).
[1] D. D. Awschalom, L. C. Bassett, A. S. Dzurak, E. L. Hu, and J. R. Petta, Science 339, 1174 (2013).

[2] S. A. Wolf et al., Science 294, 1488 (2001).

[3] C. Chappert, A. Fert, and F. N. Van Dau, Nat. Mater. 6, 813 (2007).

[4] Y. W. Son, M. L. Cohen, and S. G. Louie, Nature (London) 444, 347 (2006).

[5] W. L. Wang, O. V. Yazyev, S. Meng, and E. Kaxiras, Phys. Rev. Lett. 102, 157201 (2009).
[6] J. Fernández-Rossier and J. J. Palacios, Phys. Rev. Lett. 99, 177204 (2007).

[7] O. V. Yazyev and M. I. Katsnelson, Phys. Rev. Lett. 100, 047209 (2008).

[8] M. Wimmer, İ. Adagideli, S. Berber, D. Tománek, and K. Richter, Phys. Rev. Lett. 100, 177207 (2008).

[9] B. Trauzettel, D. V. Bulaev, D. Loss, and G. Burkard, Nat. Phys. 3, 192 (2007). 
[10] A. D. Güçlü, P. Potasz, and P. Hawrylak, Phys. Status Solidi RRL 10, 58 (2016).

[11] A. D. Güçlü, P. Potasz, O. Voznyy, M. Korkusinski, and P. Hawrylak, Phys. Rev. Lett. 103, 246805 (2009).

[12] E. H. Lieb, Phys. Rev. Lett. 62, 1201 (1989).

[13] M. Fujita, K. Wakabayashi, K. Nakada, and K. Kusakabe, J. Phys. Soc. Jpn. 65, 1920 (1996).

[14] H. Lee, Y. W. Son, N. Park, S. Han, and J. Yu, Phys. Rev. B 72, 174431 (2005).

[15] Y. W. Son, M. L. Cohen, and S. G. Louie, Phys. Rev. Lett. 97, 216803 (2006).

[16] T. C. Li and Shao-Ping Lu, Phys. Rev. B 77, 085408 (2008).

[17] F. Cervantes-Sodi, G. Csányi, S. Piscanec, and A. C. Ferrari, Phys. Rev. B 77, 165427 (2008).

[18] T. Wassmann, A. P. Seitsonen, A. M. Saitta, M. Lazzeri, and F. Mauri, Phys. Rev. Lett. 101, 096402 (2008).

[19] A. D. Güçlü, P. Potasz, and P. Hawrylak, Phys. Rev. B 88, 155429 (2013).

[20] H. U. Özdemir, A. Altintaş, and A. D. Güçlü, Phys. Rev. B 93, 014415 (2016).

[21] H. Şahin, R. T. Senger, and S. Ciraci, J. Appl. Phys. 108, 074301 (2010).

[22] O. V. Yazyev, Rep. Prog. Phys. 73, 056501 (2010).

[23] J. Jung and A. H. MacDonald, Phys. Rev. B 79, 235433 (2009).

[24] P. Potasz, A. D. Güçlü, and P. Hawrylak, Phys. Rev. B 81, 033403 (2010).

[25] H. Feldner, Z. Y. Meng, A. Honecker, D. Cabra, S. Wessel, and F. F. Assaad, Phys. Rev. B 81, 115416 (2010).

[26] G. Z. Magda, X. Jin, I. Hagymási, P. Vancsó, Z. Osváth, P. Nemes-Incze, C. Hwang, L. P. Biró, and L. Tapasztó, Nature (London) 514, 608 (2014).

[27] M. Modarresi and A. D. Güçlü, Phys. Rev. B 95, 235103 (2017).

[28] N. M. R. Peres, F. Guinea, and A. H. Castro Neto, Phys. Rev. B 73, 125411 (2006).

[29] M. V. Ulybyshev and M. I. Katsnelson, Phys. Rev. Lett. 114, 246801 (2015).

[30] O. V. Yazyev and L. Helm, Phys. Rev. B 75, 125408 (2007).

[31] J. J. Palacios, J. Fernández-Rossier, and L. Brey, Phys. Rev. B 77, 195428 (2008).

[32] W. Jaskolski, L. Chico, and A. Ayuela, Phys. Rev. B 91, 165427 (2015)

[33] H. Gonzalez-Herrero, J. M. Gomez-Rodriguez, P. Mallet, M. Moaied, J. J. Palacios, C. Salgado, M. M. Ugeda, J. Y. Veuillen, F. Yndurain, and I. Brihuega, Science 352, 437 (2016).

[34] F. Gargiulo, G. Autés, N. Virk, S. Barthel, M. Rösner, L. R. M. Toller, T. O. Wehling, and O. V. Yazyev, Phys. Rev. Lett. 113, 246601 (2014).

[35] P. Esquinazi, D. Spemann, R. Höhne, A. Setzer, K.-H. Han, and T. Butz, Phys. Rev. Lett. 91, 227201 (2003).

[36] D. Soriano, F. Munoz-Rojas, J. Fernández-Rossier, and J. J. Palacios, Phys. Rev. B 81, 165409 (2010).

[37] R. Singh and P. Kroll, J. Phys.: Condens Matter 21, 196002 (2009).

[38] A. D. Güçlü and N. Bulut, Phys. Rev. B 91, 125403 (2015).

[39] A. Altıntaş and A. D. Güçlü, Solid State Commun. 281, 44 (2018).
[40] Y. Zhang, S.-Yu. Li, H. Huang, W.-T. Li, J.-B. Qiao, W.-X. Wang, L.-J. Yin, K.-K. Bai, W. Duan, and L. He, Phys. Rev. Lett. 117, 166801 (2016).

[41] O. V. Yazyev, Phys. Rev. Lett. 101, 037203 (2008).

[42] V. M. Pereira, F. Guinea, J. M. B. Lopes dos Santos, N. M. R. Peres, and A. H. Castro Neto, Phys. Rev. Lett. 96, 036801 (2006).

[43] L. Talirz, H. Söde, T. Dumslaff, S. Wang, J. Ramon SanchezValencia, J. Liu, P. Shinde, C. A. Pignedoli, L. Liang, V. Meunier, N. C. Plumb, M. Shi, X. Feng, A. Narita, K. Müllen, R. Fasel, and P. Ruffieux, ACS Nano 11, 1380 (2017).

[44] G. D. Nguyen, H.-Z. Tsai, A. A. Omrani, T. Marangoni, M. Wu, D. J. Rizzo, G. F. Rodgers, R. R. Cloke, R. A. Durr, Y. Sakai, F. Liou, A. S. Aikawa, J. R. Chelikowsky, S. G. Louie, F. R. Fischer, and M. F. Crommie, Nat. Nanotechnol. 12, 1077 (2017).

[45] M. C. Chong, N. Afshar-Imani, F. Scheurer, C. Cardoso, A. Ferretti, D. Prezzi, and G. Schull, Nano Lett. 18, 175 (2018).

[46] D. G. de Oteyza, A. Garcia-Lekue, M. Vilas-Varela, N. MerinoDiez, E. Carbonell-Sanromá, M. Corso, G. Vasseur, C. Rogero, E. Guitián, J. Ignacio Pascual, J. Enrique Ortega, Y. Wakayama, and D. Peña, ACS Nano 10, 9000 (2016).

[47] H. Hayashi, J. Yamaguchi, H. Jippo, R. Hayashi, N. Aratani, M. Ohfuchi, S. Sato, and H. Yamada, ACS Nano 11, 6204 (2017).

[48] R. M. Jacobberger and M. S. Arnold, ACS Nano 11, 8924 (2017).

[49] R. S. Jordan, Y. L. Li, C.-W. Lin, R. D. McCurdy, J. B. Lin, J. L. Brosmer, K. L. Marsh, S. I. Khan, K. N. Houk, R. B. Kaner, and Y. Rubin, J. Am. Chem. Soc. 139, 15878 (2017).

[50] A. M. Dimiev, A. Khannanov, I. Vakhitov, A. Kiiamov, K. Shukhina, and J. M. Tour, ACS Nano 12, 3985 (2018).

[51] N. Kalashnyk, K. Mouhat, J. Oh, J. Jung, Y. Xie, E. Salomon, T. Angot, F. Dumur, D. Gigmes and S. Clair, Nat. Commun. 8, 14735 (2017).

[52] L. Chen, L. He, H. S. Wang, H. Wang, S. Tang, C. Cong, H. Xie, L. Li, H. Xia, T. Li, T. Wu, D. Zhang, L. Deng, T. Yu, X. Xie, and M. Jiang, Nat. Commun. 8, 14703 (2017).

[53] P. H. Jacobse, A. Kimouche, T. Gebraad, M. M. Ervasti, J. M. Thijssen, P. Liljeroth, and I. Swart, Nat. Commun. 8, 119 (2017).

[54] G. Schubert and H. Fehske, Phys. Rev. Lett. 108, 066402 (2012).

[55] M. Topsakal, E. Aktürk, H. Sevinçli, and S. Ciraci, Phys. Rev. B 78, 235435 (2008).

[56] Q. Q. Dai, Y. F. Zhu, and Q. Jiang, J. Phys. Chem. C 117, 4791 (2013).

[57] P. Koskinen, S. Malola, and H. Hakkinen, Phys. Rev. Lett. 101, 115502 (2008).

[58] O. Voznyy, A. D. Güçlü, P. Potasz, and P. Hawrylak, Phys. Rev. B 83, 165417 (2011).

[59] A. H. C. Neto, F. Guinea, N. M. R. Peres, K. S. Novoselov, and A. K. Geim, Rev. Mod. Phys. 81, 109 (2009).

[60] S. Reich, J. Maultzsch, C. Thomsen, and P. Ordejon, Phys. Rev. B 66, 035412 (2002).

[61] P. Potasz, A. D. Güçlü, and P. Hawrylak, Phys. Rev. B 82, 075425 (2010). 